\title{
Study on the Bactericidal Effect of Ortho-Phthalaldehyde Combination Disinfectant
}

\author{
Yi Jialin, Niu Bing, Deng Zhirui, Chen Qin* \\ School of Life Sciences, Shanghai University, Shanghai, China

\section{Email address:} \\ yjleleen@163.com (Yi Jialin), bingniu@shu.edu.cn (Niu Bing), dengzhirui@staff.shu.edu.cn (Deng Zhirui), \\ chenqincc@staff.shu.edu.cn (Chen Qin) \\ ${ }^{*}$ Corresponding author
}

\section{To cite this article:}

Yi Jialin, Niu Bing, Deng Zhirui, Chen Qin. Study on the Bactericidal Effect of Ortho-Phthalaldehyde Combination Disinfectant. Science Discovery. Vol. 5, No. 6, 2017, pp. 479-485. doi: 10.11648/j.sd.20170506.25

Received: November 14, 2017; Accepted: November 22, 2017; Published: December 28, 2017

\begin{abstract}
This study used combination of ortho-phthalaldehyde(OPA) and quaternary ammonium surfactants (QAS) with different concentration to kill Escherichia coli, Staphylococcus aureus, Candida albicans and Aspergillus niger conidia. The concentration of the combination disinfectant to kill Escherichia coli, Staphylococcus aureus and Candida albicans was 75mg/L OPA and 30mg/L QAS within 10 min, respectively. The average killing log value was above 4 when Aspergillus niger conidia was exposed in $3.75 \mathrm{~g} / \mathrm{L}$ OPA and $1.50 \mathrm{~g} / \mathrm{L}$ QAS for one hour. Then the effects of time, temperature and organic concentration on the bactericidal effect were measured. And the metal corrosion and stability of the combination disinfectant were determined. In addition, these results showed that the combination disinfectant had a high efficiency of bacteria killing and the concentration of organic affected its bactericidal effect. The average killing log value gradually increased along with the temperature within $10^{\circ} \mathrm{C}$. to $40^{\circ} \mathrm{C}$. And the combination disinfectant also has the advantages of low corrosive of metal, good stability and so on.
\end{abstract}

Keywords: Ortho-Phthalaldehyde, Escherichia coli, Metal Corrosive, Quaternary Ammonium Surfactants

\section{邻苯二甲醛类复合消毒剂杀菌效果的研究}

易佳琳，钮冰，邓志瑞，陈沁*

上海大学生命科学学院, 上海, 中国

邮箱

yjleleen@163.com (易佳琳), bingniu@shu.edu.cn（钮冰）, dengzhirui@staff.shu.edu.cn（邓志瑞）, chenqincc@staff.shu.edu.cn（陈沁）

摘要: 本研究用不同配比的邻苯二甲醛和季铵盐类阳离子表面活性剂组成复合消毒剂, 杀灭大肠杆菌、金黄色葡萄球 菌、白色念珠菌和黑曲霉分生孢子。确定了 $3.75 \mathrm{~g} / \mathrm{L}$ 邻苯二甲醛和 $1.50 \mathrm{~g} / \mathrm{L}$ 季铵盐类阳离子表面活性剂对黑曲霉分生孢子 作用 1 小时, KL大于 $4 ; 75 \mathrm{mg} / \mathrm{L}$ 邻苯二甲醛和 $30 \mathrm{mg} / \mathrm{L}$ 季铵盐类阳离子表面活性剂作用 10 分钟对大肠杆菌、金黄色葡萄 球菌和白色念珠菌可达到较好的杀灭效果。在此基础上, 进一步测定了时间、温度和有机物浓度对杀菌效果的影响以 及复合消毒剂的稳定性和金属腐蚀性。结果表明, 邻苯二甲醛类复合消毒剂具有杀菌速度快、金属腐蚀性小和稳定性 良好等优点。研究表明, 在 $10^{\circ} \mathrm{C}$ 至 $40^{\circ} \mathrm{C}$ 范围内, 杀菌效果随着温度的升高而增强。

关键词：邻苯二甲醛, 大肠杆菌, 金属腐蚀性, 季铵盐类阳离子表面活性剂 


\section{1. 引言}

消毒剂的使用历史悠久, 人类在公元前就开始利用醇 类消毒剂进行杀菌。目前, 市场常用消毒剂有过乙酸、醛 类、过氧化氢、异噻唑酮和双胍异氭尿酸酯等多种化合物 [1]。邻苯二甲醛（Ortho-Phthalaldehyde，OPA）是近年来 研制的一种新型化学消毒剂, 安全性相对较高, 对人体刺 激较小, 杀菌能力强[2]。目前国内外对OPA消毒的应用研 究已有报道, 主要研究OPA作为消毒剂是否可以代替戊二 醛 $[3,4]$ 。OPA作为一种高效消毒剂, 与戊二醛相比, 具有 广谱、高效、腐蚀性低、刺激性小、稳定性好和挥发性小 等特点 $[4,5]$ 。此外OPA对耐戊二醛的龟分枝杆菌也具有良 好的杀灭作用 $[4,6]$ 。季铵盐化合物因其高效的杀菌性能以 及良好的水溶性、稳定性、安全低毒、无不良气味、价格 便宜等诸多优点已应用到众多领域 [7-9]。将OPA与季铵盐 类阳离子表面活性剂 (quaternary ammonium surfactants, QAS）进行复配来提高消毒剂的杀菌能力, 减少两类消毒 剂的用量, 克服部分单方使用时的缺点。鉴于此, 本研究 拟将OPA与 $\mathrm{QAS}$ 复配使用, 研究两种消毒剂不同配比对杀 菌效果的影响, 并对复合消毒剂的稳定性和金属腐蚀性等 进行了研究。

\section{2. 材料和方法}

\section{1. 材料}

大肠杆菌（ATCC 8099）、金黄色葡萄球菌（ATCC 6538）、白色念珠菌（ATCC 10231）和黑曲䨋菌（ATCC 16404 ) 由上海出入境检验检疫局提供。邻苯二甲醛购自 国药集团化学试剂有限公司, 季铵盐类阳离子表面活性剂
购自阿拉丁试剂有限公司。铜片（GB2060）、铝片 (GB11173) 、不锈钢片（GB1220）和碳钢片（GB700） 均购自金坛市振兴实验器材厂。

\section{2. 方法}

\subsection{1. 细菌繁殖体悬液和真菌悬液的制备}

取第3 6代的营养琼脂培养基（细菌）或沙堡琼脂培 养基（真菌）培养 18 小时 24小时的新鲜培养物, 吸取 $3.0 \mathrm{~mL} \sim 5.0 \mathrm{~mL}$ 胰蛋白胨生理盐水溶液（trypyone saline， TPS）加入培养血内, 反复吹吸, 洗下菌苔, 将洗液移至 另一无菌试管中, 震荡均匀后测定菌悬液的 $\mathrm{OD}_{600}$ 吸光度 值, 用TPS将菌悬液稀释到浓度为 $1 \times 10^{8} \sim 5 \times 10^{8}$ (细菌) 或 $1 \times 10^{7} \sim 5 \times 10^{7}$ (真菌)。

\subsection{2. 黑曲霉分生孢子悬液的制备}

取第3代麦芽浸膏肉汤培养基培养的新鲜培养物, 接 种于罗氏瓶, $30^{\circ} \mathrm{C}$ 培养 42 小时 48 小时。生理盐水 (含体 积比 $0.05 \%$ 的吐温 $80 ）$ 清洗并于装有玻璃珠的三角瓶中轻 轻振摇 1 分钟, 滤过菌丝, 该悬液在 $2^{\circ} \mathrm{C} \sim 8^{\circ} \mathrm{C}$ 储存不超过 2 天。

\subsection{3. 中和剂鉴定}

中和剂为 $1 \%$ 亚硫酸钠 $+1 \%$ 卵磷脂 $+7 \%$ 吐温 80 的磷酸 盐缓冲溶液, 实验分组见表 1 。吸取表1中各组作用后的混 合溶液 $1 \mathrm{~mL}$ 接种于平板, 作活菌培养计数, 若平板生长菌 落数超过 300 个, 用TPS适宜稀释后再进行活菌培养计数。

表1 中和剂鉴定分组。

\begin{tabular}{lll}
\hline 分组 & 试剂 & 目的 \\
\hline 1 & 中和剂+无菌水+菌悬液 & 观察中和剂是否抑菌 \\
2 & (复合消毒剂+中和剂) +菌悬液 & 观察中和产物对细菌的生长繁殖是否有影响 \\
3 & 稀释液TPS+无菌水+菌悬液 & 菌数对照 \\
4 & 稀释液TPS+无菌水+中和剂 & 阴性对照 \\
\hline
\end{tabular}

$$
\text { 组间菌落数误差率 }=\left[\frac{(\text { 三组间菌落平均数 }- \text { 各组菌落平均数 }) \text { 的绝对值之和 }}{\text { 三组菌落平均数之和 }}\right] \times 100 \% \text { 。 }
$$

第 $1 、 2$ 和 3 组有相似量试验菌生长, 其组间菌落数误 差率应不超过 $15 \%$ ，第4组无菌生长。

\subsection{4. 悬液定量杀菌实验}

用无菌硬水配制消毒剂, 菌悬液与 $3 \% \mathrm{BSA}$ 按 $1: 1$ 混合 作用 5 分钟, 无菌试管内加入 $1.0 \mathrm{~mL}$ 上述混合液与 $4.0 \mathrm{~mL}$ 消 毒剂 (对照组为等量无菌硬水), 迅速混匀并计时, 作用 至规定时间后, 分别吸取 $0.5 \mathrm{~mL}$ 菌药混合液, 加入含 $4.5 \mathrm{~mL}$ 中和剂的无菌试管内吹打均匀, 作用 10 分钟后进行梯度稀 释, 接种于无菌培养皿, 倒入培养基并摇勺, 进行培养计 数, 并计算其杀灭对数值（killing log value, KL）, 各实 验均重复3次 $[10]$ 。

$$
\begin{array}{r}
\mathrm{KL}=\text { 对照组平均活菌浓度的对数值 }\left(N_{0}\right) \\
- \text { 实验组活菌浓度对数值 }\left(N_{x}\right)
\end{array}
$$

2.2.5. 温度、作用时间和有机物对复合消毒剂作用效果 的影响

用含 $75 \mathrm{mg} / \mathrm{L} \mathrm{OPA}$ 和 $30 \mathrm{mg} / \mathrm{L} \mathrm{QAS}$ 的复合消毒剂对大 肠杆菌作用不同时间 (3分钟、5 分钟、10分钟和 20分钟) 、 不同温度 $\left(10^{\circ} \mathrm{C} 、 20^{\circ} \mathrm{C} 、 30^{\circ} \mathrm{C}\right.$ 和 $\left.40^{\circ} \mathrm{C}\right)$ 和不同有机物浓度 （将大肠杆菌悬液与无菌小牛血清配制成含 $0 \% 、 5 \% 、 25 \%$ 和 $50 \%$ 小牛血清的菌悬液）, 计算其杀灭对数值, 各组实 验均重复3次。 


\subsection{6. 金属腐蚀性实验}

将不锈钢、碳钢、铜和铝片, 经打磨、洗净、干燥后 称重, 然后浸泡在消毒液中 72 小时后, 取出金属片洗净, 去腐蚀物, 干燥后再次称重。观察与记录金属片颜色变化, 每种金属取3次试验平均值并计算金属腐蚀速率，判断腐 蚀级别[11]。

$$
\mathrm{R}=\frac{\left[8.76 \times 10^{7} \times\left(\mathrm{m}-\mathrm{m}_{\mathrm{t}}-\mathrm{m}_{\mathrm{k}}\right)\right]}{\mathrm{S} \times \mathrm{t} \times \mathrm{d}}
$$

式中 $\mathrm{m}$ 为实验前金属片重量 $(\mathrm{g}) ; \mathrm{m}_{\mathrm{t}}$ 为实验后金属 片重量 $(\mathrm{g}) ; \mathrm{m}_{\mathrm{k}}$ 为化学处理去除腐蚀产物样片失重值 $(\mathrm{g})$; $\mathrm{S}$ 为金属片的表面积总值 $\left(\mathrm{cm}^{2}\right)$; $\mathrm{t}$ 为实验时间 $(\mathrm{h}) ; \mathrm{d}$ 为金属材料密度 $\left(\mathrm{kg} / \mathrm{m}^{3}\right)$ 。分级标准为: $R$ 小于 0.0100 为 基本无腐蚀 $(\mathrm{A}) ; 0.0100 \sim 0.100$ 为轻度腐蚀 $(\mathrm{B}) ; 0.100 \sim 1.00$ 为中度腐蚀 $(\mathrm{C})$; 大于 1.00 为重度腐蚀 $(\mathrm{D})$ 。

\subsection{7. 稳定性}

配制浓度为 $7.5 \mathrm{~g} / \mathrm{L}$ OPA和 $3 \mathrm{~g} / \mathrm{L}$ QAS的复合消毒剂于 $54^{\circ} \mathrm{C}$ 放置 14 天, 分别测定 0 天和 14 天时消毒剂中有效成分 含量, 计算其下降率。

OPA含量的测定: 取邻苯二甲醛复合消毒剂适量于碘 量瓶, 加入蒸馏水 $30 \mathrm{~mL} 、 10 \% \mathrm{NaHSO}_{3} 1 \mathrm{~mL}$, 静置反应 15 分钟后, 加入 $50 \mathrm{~mL}$ 蒸馏水和 $2 \mathrm{ml}$ 淀粉指示剂, 用 $0.1 \mathrm{~mol} / \mathrm{L}$ 碘标准溶液滴定, 出现兰色止, 不计算消耗碘溶液量。然 后加入 $10 \mathrm{~mL} 10 \% \mathrm{Na}_{2} \mathrm{CO}_{3}$, 立刻用 $0.05 \mathrm{~mol} / \mathrm{L}$ 碘标准溶液滴 定, 出现兰色 $5 \mathrm{~s}$, 记录消耗的碘溶液用量, 计算 $\mathrm{OPA}$ 含量。 OPA 含量 $\%=0.05 \times 0.067065 \times \mathrm{V} \times 100 \% \quad(\mathrm{~V}$ 为 $0.05 \mathrm{~mol} / \mathrm{L}$ 碘溶液的用量 $\mathrm{mL}$ )。

QAS含量的测定: 取液体样品适量体积, 置 $250 \mathrm{~mL}$ 碘 量瓶中。加蒸馏水 $50 \mathrm{~mL}$ 与氢氧化钠试液 $1 \mathrm{~mL}$, 摇匀。再 加溴酚蓝指示液 $0.4 \mathrm{~mL}$ 与氯仿 $10 \mathrm{~mL}$ 。用四苯硼钠滴定液滴
定, 边滴边摇匀, 待氯仿层的蓝色消失, 记录四苯硼钠滴 定液用量, 同时做空白实验。计算公式为

$$
\rho(g / L)=\frac{c \times V_{s t} \times 0.3400}{V} \times 1000
$$

式中c为四苯硼钠滴定液的浓度 $(\mathrm{mol} / \mathrm{L}) ; \mathrm{V}_{\mathrm{st}}$ 为四苯 硼钠滴定液样品与空白体积差 $(\mathrm{mL}) ; \mathrm{V}$ 为碘量瓶中含 $\mathrm{QAS}$ 原液体积 $(\mathrm{mL})$ 。

\subsection{8. 集装箱表面自然菌消毒实验}

抽取未经消毒处理以及清洗的标准集装箱空箱 4 个, 取口岸常用消毒剂 $\mathrm{U}$ 和不同浓度的复合消毒剂, 用喷雾器 进行表面喷酒，作用时间 10 分钟，用标准无菌规格板 $(5 \mathrm{~cm} \times 5 \mathrm{~cm})$ 在箱内表面使用无菌棉拭子进行集装箱箱面 的采样。采样前将无菌棉拭子在含有 $3 \mathrm{~mL}$ 无菌生理盐水试 管中浸湿, 挤干, 对无菌规格板框定的消毒剂或无菌水喷 酒过的物体表面涂抹采样, 再将棉拭子放入含中和剂的无 菌试管, 震荡混匀 10 分钟后进行培养计数, 并计算其杀灭 率。

$$
\text { 杀灭率 }=\frac{\text { 对照组存活菌量 }- \text { 消毒剂处理组存活菌量 }}{\text { 对照组存活菌量。 }}
$$

\section{3. 结果}

\section{1. 中和剂的鉴定}

中和剂鉴定实验中, 大肠杆菌菌落数的组间误差率为 $6.79 \%$, 白色念珠菌为 $4.93 \%$, 黑曲霉分生孢子为 $12.50 \%$, 均不超过 $15 \%$, 符合消毒规范要求 [12] (表 2)。由此可知, 该中和剂可有效终止复合消毒剂对大肠杆菌、金黄色葡萄

\begin{tabular}{|c|c|c|c|c|}
\hline \multirow{3}{*}{ 组别 } & \multirow{3}{*}{ 试剂 } & 大肠杆菌 & 白色念珠菌 & 黑曲電分生孢子 \\
\hline & & 菌落数 & 菌落数 & 菌落数 \\
\hline & & $(\mathrm{cfu} / \mathrm{mL})$ & $(\mathbf{c f u} / \mathbf{m L})$ & $(\mathrm{cfu} / \mathbf{m L})$ \\
\hline 1 & 中和剂+无菌水+菌悬液 & 1442 & 1326 & $9.0 \times 10^{4}$ \\
\hline 2 & (复合消毒剂 + 中和剂) + 菌悬液 & 1260 & 1157 & $6.5 \times 10^{4}$ \\
\hline 3 & 稀释液TPS+无菌水+菌悬液 & 1507 & 1266 & $8.5 \times 10^{4}$ \\
\hline 4 & 稀释液TPS+无菌水+中和剂 & 0 & 0 & 0 \\
\hline
\end{tabular}
球菌、白色念珠菌和黑曲霉分生孢子的杀灭的作用。

表2 中和剂的鉴定。

\section{2. 复合消毒剂杀菌效果的检测}

\subsection{1. 复合消毒剂对大肠杆菌的杀灭作用}

在悬液定量杀菌实验中，改变消毒剂的组分会影响大肠杆菌的杀灭效果，当OPA和QAS浓度分别为 $300 \mathrm{mg} / \mathrm{L} （$ 图 $1 \mathrm{~A}$ ) 和 $100 \mathrm{mg} / \mathrm{L}$ (图 $1 \mathrm{~B}$ ) 时, 杀菌效果达到消毒规范要求 $[12](\mathrm{KL}>5$ )。在此基础上, 将两种消毒剂进行不同浓度复配。 当复合消毒剂为 $75 \mathrm{mg} / \mathrm{L} \mathrm{OPA}$ 和 $30 \mathrm{mg} / \mathrm{L}$ QAS (图 $1 \mathrm{C}$ ) 时, $\mathrm{KL}$ 达到 5.71 , 杀灭效果同 $300 \mathrm{mg} / \mathrm{L} \mathrm{OPA}$ 和 $100 \mathrm{mg} / \mathrm{L} \mathrm{QAS}$ 相当, 故 $25 \%$ 的OPA和 $30 \%$ 的QAS复配即可达到单方消毒剂的杀菌效果, 降低了消毒剂的使用浓度, 两种消毒剂复合使用增 强了其杀菌效果。 

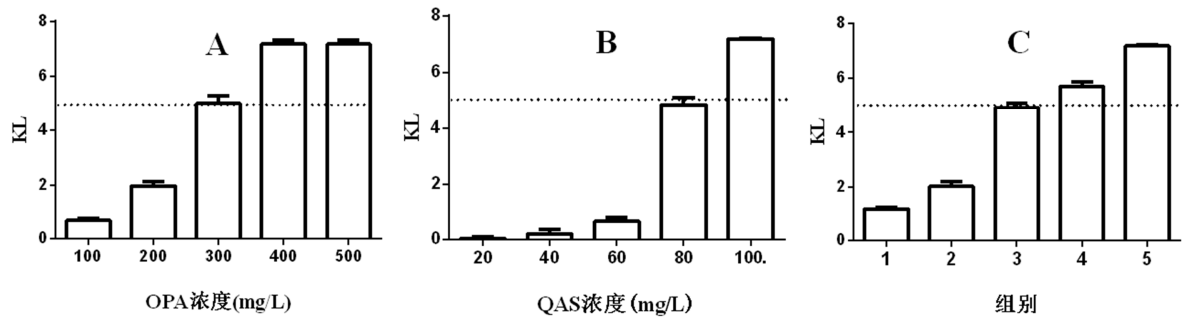

图1 不同消毒剂对大肠杆菌的杀灭作用.

注: 图1C中组别1 5消毒剂浓度分别为: $7.5 \mathrm{mg} / \mathrm{L} \mathrm{OPA}+5 \mathrm{mg} / \mathrm{L}$ QAS; $37.5 \mathrm{mg} / \mathrm{L} \mathrm{OPA}+15 \mathrm{mg} / \mathrm{L} \mathrm{QAS} ; 37.5 \mathrm{mg} / \mathrm{L} \mathrm{OPA}+25 \mathrm{mg} / \mathrm{L} \mathrm{QAS} ; 75 \mathrm{mg} / \mathrm{L} \mathrm{OPA}+30 \mathrm{mg} / \mathrm{L}$ QAS; $75 \mathrm{mg} / \mathrm{L} \mathrm{OPA}+50 \mathrm{mg} / \mathrm{L} \mathrm{QAS}$; 作用时间为 10 分钟。

\subsection{2. 复合消毒剂对其它菌的杀灭作用}

我们用复合消毒剂对金黄色葡萄球菌、白色念珠菌 和黑曲霉分生孢子进行悬液定量杀菌实验, 结果显示 $75 \mathrm{mg} / \mathrm{L} \mathrm{OPA}$ 和 $30 \mathrm{mg} / \mathrm{L}$ QAS复合使用，作用 10 分钟对金 黄色葡萄球菌和白色念珠菌的杀灭效果即可满足消毒规
范要求 $[12], \mathrm{KL}$ 分别达到 5.10 和 4.22 。3.75g/L OPA和 $1.50 \mathrm{~g} / \mathrm{L}$ QAS对黑曲霸分生孢子作用 1 小时, KL大于 4 。由 上述结果可知复合消毒剂对革兰氏阴性菌、革兰氏阳性 菌、真菌和黑曲霉分生孢子都具有较好的杀灭作用，复 合消毒剂杀菌效果好，杀菌速度快。
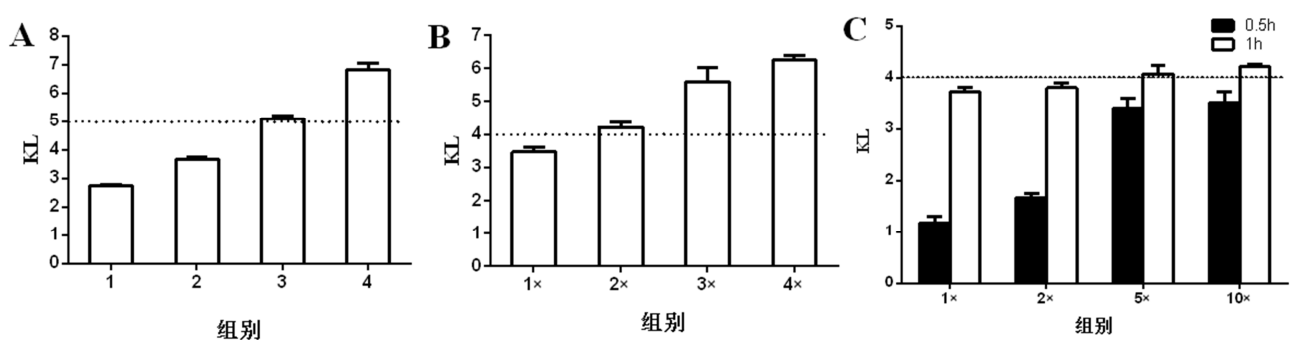

图2 复合消毒剂对金黄色葡萄球菌、白色念珠菌和黑曲霉分生孢子的杀灭作用.

注: 图2A中组别1 4消毒剂浓度分别为: $37.5 \mathrm{mg} / \mathrm{L} \mathrm{OPA}+15 \mathrm{mg} / \mathrm{L} \mathrm{QAS} ; 37.5 \mathrm{mg} / \mathrm{L} \mathrm{OPA}+25 \mathrm{mg} / \mathrm{L} \mathrm{QAS} ; 75 \mathrm{mg} / \mathrm{L} \mathrm{OPA}+30 \mathrm{mg} / \mathrm{L} \mathrm{QAS} ; 75 \mathrm{mg} / \mathrm{L} \mathrm{OPA}+50 \mathrm{mg} / \mathrm{L}$ QAS; 实验菌株为金黄色葡萄球菌。图 $2 \mathrm{~B}$ 中 $1 \times$ 消毒剂浓度为: $37.5 \mathrm{mg} / \mathrm{L} \mathrm{OPA}+15 \mathrm{mg} / \mathrm{L}$ QAS; 实验菌株为白色念珠菌。图 $2 \mathrm{C}$ 中 $1 \times$ 消毒剂浓度为: $0.75 \mathrm{~g} / \mathrm{L}$ $\mathrm{OPA}+0.30 \mathrm{~g} / \mathrm{L} \mathrm{QAS}$; 实验菌株为黑曲霉分生孢子。

\section{3. 外界条件对复合消毒剂杀菌效果的影响}

\section{3. 1. 有机物对复合消毒剂杀菌效果的影响}

我们分别配制了含 $0 \% 、 5 \% 、 25 \%$ 和 $50 \%$ 有机物（小 牛血清) 的菌悬液进行杀菌实验, 结果如图3所示。有机 物对复合消毒剂杀菌效果有一定的干扰, 小牛血清含量为 $0 \%$ 时, KL为 7.26 ; 有机物含量为 $5 \% 、 25 \%$ 和 $50 \%$ 时, $\mathrm{KL}$ 值分别为 5.88 、 5.40 和 4.33 , 杀菌效果略有下降。当有机 物含量为 $25 \%$ 时, KL大于 5 , 仍然能有效杀灭大肠杆菌。

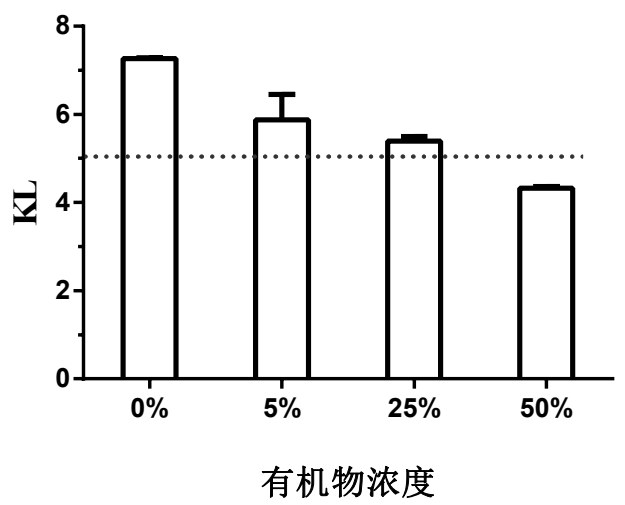

图3 有机物浓度对复合消毒剂杀菌效果的影响。

\section{3. 2. 温度对复合消毒剂杀菌效果的影响}

在温度对复合消毒剂杀菌效果影响的实验中, 随着温 度的升高, 复合消毒剂杀菌效果增强。消毒剂在 $10^{\circ} \mathrm{C}$ 时杀 菌能力最弱, 作用 10 分钟时的KL为 4.29 左右。温度上升到 $30^{\circ} \mathrm{C}, \mathrm{KL}$ 为 5.16 , 当温度上升为 $40^{\circ} \mathrm{C}$ 时, $\mathrm{KL}$ 达到6.29。故 温度对复合消毒剂杀菌效果有一定的影响, 温度越高杀菌 效果越好。

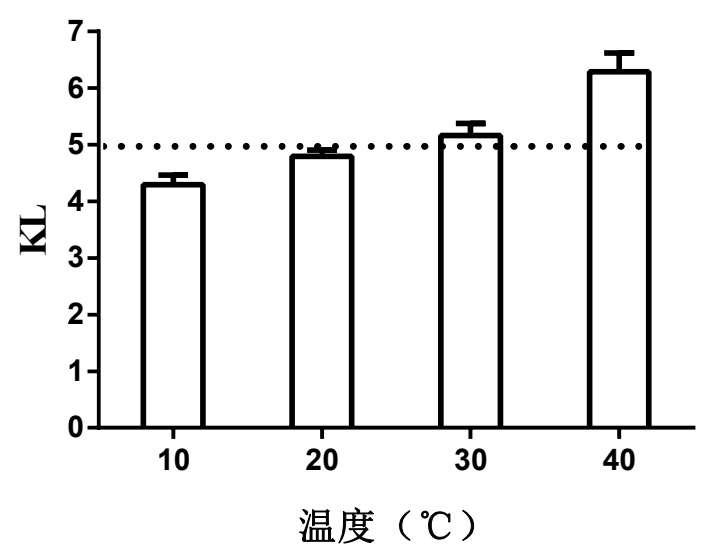

图4 温度对复合消毒剂杀菌效果的影响。 


\section{3. 3. 时间对复合消毒剂杀菌效果的影响}

我们使用含 $75 \mathrm{mg} / \mathrm{L}$ OPA和 $30 \mathrm{mg} / \mathrm{L}$ QAS的复合消毒 剂, 作用时间为 3 分钟 $\sim 20$ 分钟 (图5)。复合消毒剂随着 杀菌时间的延长，KL从5.14升高到6.90。复合消毒剂在 3 分钟时杀灭对数值就已大于 5 , 杀菌作用快速, 效果优异。

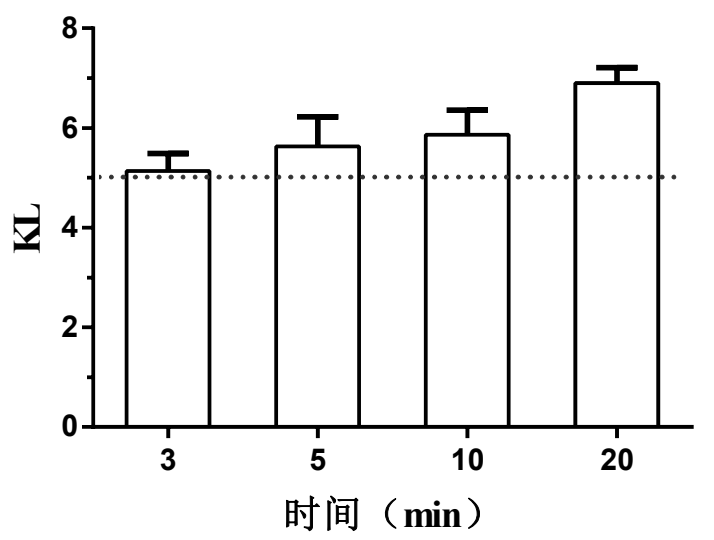

图5 作用时间对复合消毒剂杀菌效果的影响。

\section{4. 复合消毒剂的应用}

消毒剂处理集装箱空箱后, 经活菌培养计数并计算消 毒剂的杀灭率, 结果如表3所示。由表可知, 复合消毒剂 对空箱表面的杀菌效果随浓度的升高而增强。10倍浓度下 的杀菌率高于口岸常用消毒剂 $\mathrm{U}$ 。浓度为 $750 \mathrm{mg} / \mathrm{L} \mathrm{OPA}$ 和
$300 \mathrm{mg} / \mathrm{L}$ QAS的复合消毒剂对空箱表面自然菌杀灭率即 可达到 $79.3 \%$ 。

表3 复合消毒剂对集装箱表面自然菌的杀灭率（\%）。

\begin{tabular}{|c|c|c|c|c|}
\hline \multirow{2}{*}{ 组别 } & \multicolumn{3}{|c|}{ 75mg/L OPA+30mg/L QAS $(1 \times)$} & \multirow{2}{*}{$\mathbf{U}$} \\
\hline & $1 \times$ & $5 \times$ & $10 \times$ & \\
\hline 左侧 & 26.9 & 52 & 82.3 & 73.1 \\
\hline 后侧 & 65.0 & 76.8 & 84.6 & 85.5 \\
\hline 右侧 & 53.1 & 60.0 & 70.7 & 64.1 \\
\hline 平均值 & 48.3 & 62.9 & 79.3 & 74.3 \\
\hline
\end{tabular}

\section{5. 复合消毒剂的金属腐蚀性实验}

碳钢片、铜片、不锈钢片和铝片在经过不同浓度的复 合消毒剂浸泡72小时后外观变化如图6所示。经过复合消 毒剂浸泡的铝片、铜片和不锈钢片表面没有明显的锈斑, 铜片随浓度升高, 颜色变浅, 而经过浸泡的碳钢片表面部 分被腐蚀。将金属片在浸泡前和浸泡后分别称重, 计算出 复合消毒剂对金属片的腐蚀速率（表3）。复合消毒剂浓 度越高, 对金属片的腐蚀性越大。通过比较4种经消毒剂 浸泡过的金属片可以看出, 金属片材质不同, 消毒剂对其 的腐蚀程度也不同。在高浓度复合消毒剂 $7.5 \mathrm{~g} / \mathrm{L} \mathrm{OPA}$ 和 $3.0 \mathrm{~g} / \mathrm{L} \mathrm{QAS}$ 处理后, 铝片基本无腐蚀; 高浓度复合消毒剂 对碳钢、铜和不锈钢浸泡72小时后只有轻度腐蚀。

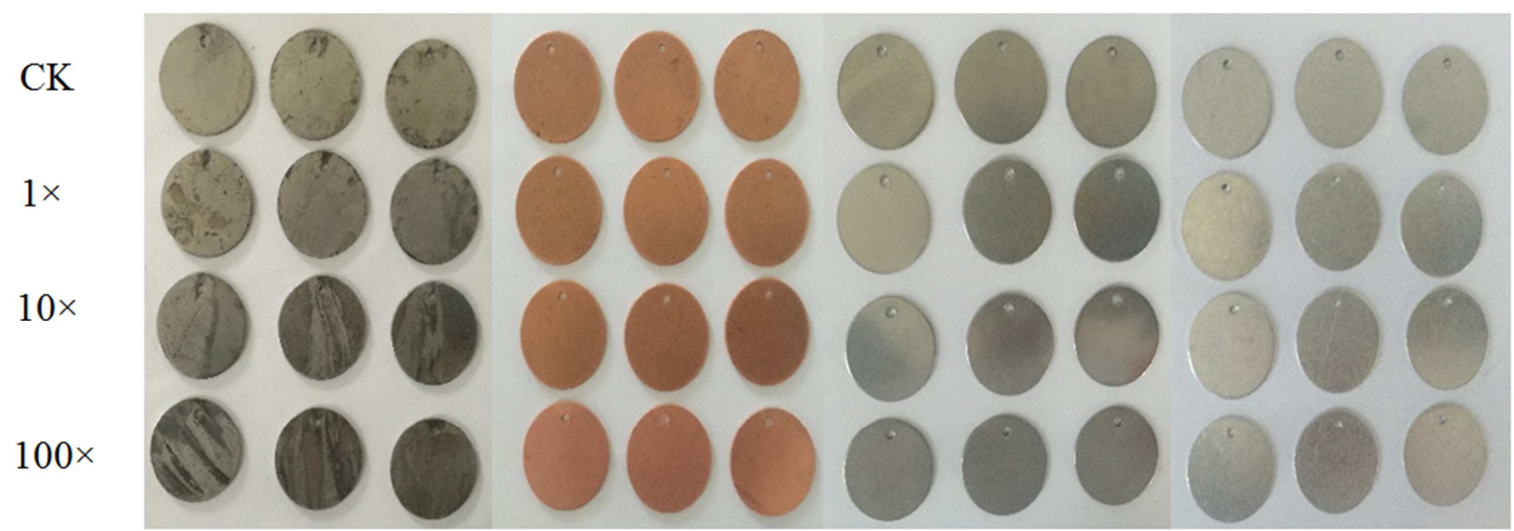

注：图6中金属分别为碳钢、铜、不锈钢和铝

图6 复合消毒剂处理后金属片外观变化。

表4 复合消毒剂金属腐蚀性检测。

\begin{tabular}{llll}
\hline 金属类型 & \multicolumn{1}{c}{ 腐蚀速率R } & $\mathbf{1 0 0 \times}$ \\
\cline { 2 - 4 } & $\mathbf{1 \times}$ & $\mathbf{1 0} \times$ & $0.0933 \pm 0.0692(\mathrm{~B})$ \\
碳钢 & $0.0835 \pm 0.0045(\mathrm{~B})$ & $0.0907 \pm 0.0178(\mathrm{~B})$ & $0.0158 \pm 0.0044(\mathrm{~B})$ \\
铜 & $0.0008 \pm 0.0003(\mathrm{~A})$ & $0.0015 \pm 0.0015(\mathrm{~A})$ & $0.0589 \pm 0.0520(\mathrm{~B})$ \\
铝 & $0.0297 \pm 0.0097(\mathrm{~B})$ & $0.0440 \pm 0.0186(\mathrm{~B})$ & $0.0061 \pm 0.0072(\mathrm{~A})$ \\
\hline
\end{tabular}

注: $1 . \mathrm{A} 、 \mathrm{~B}$ 分别为基本无腐蚀, 轻度腐蚀。2. 将 $75 \mathrm{mg} / \mathrm{L} \mathrm{OPA}+30 \mathrm{mg} / \mathrm{L}$ QAS定为 $1 \times$ 浓度。

\section{6. 复合消毒剂稳定性测定}

测定在 $54^{\circ} \mathrm{C}$ 条件下保存 0 天和 14 天后复合消毒剂中 OPA和QAS的含量, 结果如图4所示: 放置14天后, OPA
含量下降 $11.84 \%$, QAS含量下降了 $9.33 \%$ 。复合消毒剂 $54^{\circ} \mathrm{C}$ 放置14天稳定性良好，下降率均不超过 $15 \%$ 。 


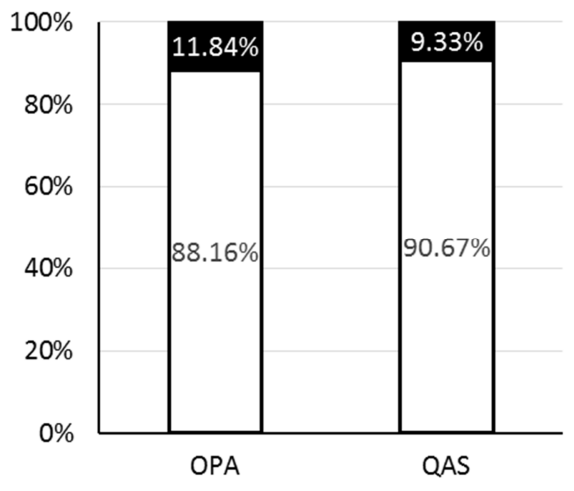

图7 复合消毒剂稳定性测定。

\section{4. 讨论}

季铵盐类消毒剂通过吸附于菌体表面，其疏水基团 逐渐渗入细胞的类脂层, 改变细胞壁和细胞膜的通透性, 使胞内容物泄露, 酶或蛋白质变性, 从而导致菌体的死 亡。季铵盐类消毒剂使用范围广, 价格便宜, 但属低效 消毒剂, 其作用范围有一定的局限性。研究证明, 季铵 盐类消毒剂对真菌、结核杆菌、亲水病毒和细菌芽狍作 用效果差[13]。文献报道OPA主要作用于菌体细胞内的核 糖体, 且还可与甘氨酸、赖氨酸和组氨酸之间发生交联 反应, 使菌体细胞内多肽链或蛋白质变性, 从而起到杀 菌的作用[14]。OPA价格较为昂贵, 将两种消毒剂进行复 配, 以增强复合消毒剂的杀菌效果降低使用成本。OPA 和QAS复配使用与单方消毒剂相比, 达到相同杀菌效果 所需的浓度都更低。KL大于 5 时, 单方消毒剂作用大肠 杆菌的浓度为 $300 \mathrm{mg} / \mathrm{L} \mathrm{OPA}$ 或 $100 \mathrm{mg} / \mathrm{L}$ QAS, 而复配使 用OPA和QAS 的浓度分别是单独使用时浓度的 $25 \%$ 和 $30 \%$, 降低了消毒剂的使用浓度, 增强了杀菌效果, 降 低了成本。同时降低OPA的用量也减轻了直接接触消毒 剂时对人体的刺激性和对皮肤的着色。复合消毒剂对真 菌和黑曲需分生狍子具有较好的杀灭效果, 浓度和作用 时间分别为 $75 \mathrm{mg} / \mathrm{L} \mathrm{OPA}+30 \mathrm{mg} / \mathrm{L}$ QAS, 作用 10 分钟和 $3.75 \mathrm{~g} / \mathrm{L} \mathrm{OPA}+1.50 \mathrm{~g} / \mathrm{L} \mathrm{QAS}$, 作用 1 小时, KL即可大于 4 , 相较单方季铵盐类消毒剂对真菌和黑曲霉分生狍子的作 用效果有了大幅度提升。消毒剂在使用过程中, 当外界 条件改变时, 复合消毒剂的作用效果也会发生变化。本 实验中杀菌时间越长, 杀菌效果越好, 可能是由于消毒 剂与细菌接触的时间越长, 进入细菌的消毒剂也会更多, 因此杀菌的效果就越好。此外, 当环境温度升高时, 消 毒剂的杀菌效果也越好, 可能是升高温度可以增强季铵 盐类消毒剂的作用效果[15], 或由于较高温度下, 消毒剂 对细菌的渗透能力较强导致。消毒现场通常会遇到各种 有机干扰物, 如分泌物、脓液、饲料残渣及粪便等, 这 些有机物可能会吸附消毒剂, 其中的有机基团也有可能 与消毒剂反应, 从而影响消毒剂的杀菌效果 [16]。本研究 中 $50 \%$ 的小牛血清使消毒剂的作用效果下降了 $40 \%, 25 \%$ 的小牛血清对杀菌效果有所抑制, 但KL仍大于 5 , 这与 王长德 [17]等的结果相一致。因此复合消毒剂不适用于有 机物含量过高的环境, 或可增加消毒剂的浓度来达到更
好消毒效果。在现场杀菌条件下, 消毒效果受到多种因 素的影响, 如环境温度、湿度、光照、风力等, 从而影 响消毒效果 [18-20]。在现场杀菌实验中，750mg/L $\mathrm{OPA}+300 \mathrm{mg} / \mathrm{L}$ QAS对空箱的杀灭率即可达到 $79.3 \%$, 高 于口岸常用消毒剂U。除了杀菌效果, 复合消毒剂的稳定 性和金属腐蚀性也是在研制、和应用中需要关注的问题。 目前口岸广泛使用的消毒剂多为化学消毒剂, 它具有杀 菌范围广、速度快、易挥发和便捷等特点, 但有些化学 消毒剂金属腐蚀性强，从而限制了其使用。复合消毒剂 的金属腐蚀性结果表明，在测试的 3 个浓度下，对铝基本 无腐蚀; 高浓度的复合消毒剂对碳钢、铜和不锈钢也只 有轻度腐蚀, 复合消毒剂腐蚀性小, 即使杀灭大肠杆菌 浓度的 100 倍, 对 4 种金属的腐蚀性最高也只有轻度腐蚀, 并且常用的集装箱为铝合金，在硬度和抗腐蚀性上均优 于钢材质，腐蚀性的结果表明该复合消毒剂对集装箱基 本无腐蚀, 可用于码头集装箱消毒。将复合消毒剂于 $54^{\circ} \mathrm{C}$ 储存 14 天后OPA的含量下降了 $11.84 \%$, QAS的含量下降 了 $9.33 \%$, 两种主要成分下降率均不超过 $15 \%$, 稳定性良 好。

\section{5. 结论}

我们通过将不同浓度的OPA和QAS复配进行悬液定 量杀菌实验, 结果显示有效杀灭大肠杆菌、金黄色葡萄球 菌和白色念珠菌的浓度为 $75 \mathrm{mg} / \mathrm{L} \mathrm{OPA}+30 \mathrm{mg} / \mathrm{L} \mathrm{QAS}$, 作 用时间为 10 分钟。有效杀灭黑曲霉分生孢子的浓度为 $3.75 \mathrm{~g} / \mathrm{L} \mathrm{OPA}+1.50 \mathrm{~g} / \mathrm{L} \mathrm{QAS}$, 作用时间为 1 小时。复合消毒 剂的杀菌效果随着时间的延长、温度的升高而升高, 随着 有机物含量的升高而下降。用不同浓度的复合消毒剂对 4 种不同金属进行金属腐蚀性实验, 发现复合消毒剂对金属 腐蚀性低, 在稳定性实验中复合消毒剂稳定性良好。

\section{致谢}

本文为国家科技支撑计划（2013BAD12B06）；国家 重点研究发展项目（2016YFD0501101）的阶段性成果之 一。

\section{参考文献}

[1] Russell AD. Introduction of biocides into clinical practice and the impact on antibiotic - resistant bacteria [J]. Journal of Applied Microbiology 2002; 92(31): 121S-135S.

[2] Martínezcamacho AP, Cortezrocha MO, Ezquerrabrauer JM, et al. Chitosan composite films: thermal, structural, mechanical and antifungal properties [J]. Carbohydrate Polymers 2010; 82: 305-15.

[3] Rutala WA, Weber DJ. New disinfection and sterilization methods [J]. Emerging Infectious Diseases 2001;7:348.

[4] 常文军, 蔡全才, 徐荷等. 邻苯二甲醛消毒剂研究进展 [J]. 中国消毒学杂志, 2004;21:67-9。 
[5] 李崇珍. 内窥镜灭菌技术进展 [J]. 护理研究, 2004;18:1799-801。

[6] 韩菲. 复方邻苯二甲醛纳米乳的研制[D]：西北农林科技大 学; 2009。

[7] 梁克中. 水处理剂简介[J]. 山东化工, 2004:37-9。

[8] 汪坤, 海洪, 金文英等. 微波辅助提取蚕沙中叶绿素的工艺 研究 $[J]$. 食品科学, 2009;30:76-9。

[9] 薛文青. 季铵盐类阳离子表面活性消毒剂研究进展 $[J]$. 中华 医院感染学杂志, 2002,12(8):79-81。

[10] Lin W, Xiao G, Jing C, et al. Bactericidal mechanism of glutaraldehyde-didecyldimethylammonium bromide as a disinfectant against Escherichia coli [J]. Journal of Applied Microbiology 2017;122:676-85.

[11] 李小林, 关潇, 林文淑等. 新型戊二醛-癸甲溴铵复合消毒 剂的杀菌效果及其影响因素的研究 $[\mathrm{J}]$. 中国国境卫生检疫 杂志, 2017(4):283-289。

[12] 卫生部卫生法制与监督司. 消毒技术规范[S]. 北京: 卫生部, 2008:135-137。

[13] 梁建生. 季铵盐类消毒剂及其应用[J]. 中国消毒学杂志, 2012, 29(2):129-131。
[14] Namba Y, Suzuki A, Takeshima N, et al. Comparative study of bactericidal activities of six different disinfectants [J]. Nagoya Journal of Medical Science, 1985, 47(3-4):101.

[15] 沈伟, 孙玉卿, 谢星辉等. 戊二醛增效剂探索 [J]. 现代预防 医学，1997:262-4。

[16] 房军, 姚楚水. 戊二醛消毒液杀菌效果及其影响因素的实验 研究 [J]. 中国消毒学杂志, 2007;24:321-4。

[17] 王长德, 张文福, 芦懿等. 邻苯二甲醛杀菌效果影响因素的 研究[J]. 中国消毒学杂志, 2004;21:182-5。

[18] Bolton N F, Cromar N J, Hallsworth P, et al. A review of the factors affecting sunlight inactivation of micro-organisms in waste stabilisation ponds: preliminary results for enterococci [J]. Water Science \& Technology A Journal of the International Association on Water Pollution Research, 2010, 61(4):885-90.

[19] Kollu K, Ormeci B. Effect of particles and bioflocculation on ultraviolet disinfection of Escherichia coli [J]. Water Research, 2012, 46(3):750-60.

[20] Nicholas R, Dunton P, Tatham A, et al. The effect of ozone and open air factor on surface-attached and biofilm environmental Listeria monocytogenes [J]. Journal of Applied Microbiology, 2013, 115(2):555-64. 